\title{
A preliminary study on the construction of double suicide gene delivery vectors by mesenchymal stem cells and the in vitro inhibitory effects on SKOV3 cells
}

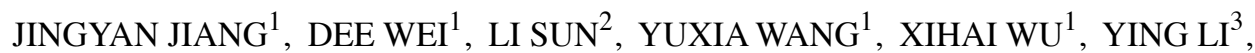 \\ ZHENGHUI FANG $^{1}$, HUI SHANG ${ }^{1}$ and ZENGTAO WEI ${ }^{1}$
}

\author{
${ }^{1}$ Department of Gynecology, Jinan Central Hospital Affiliated to Shandong University, Jinan, Shandong 250014; \\ ${ }^{2}$ Department of Gynecologic Oncology, Shandong Cancer Hospital, Jinan, Shandong 250117; Shandong Academy \\ of Medical Sciences; Shandong University, Jinan, Shandong 250012; ${ }^{3}$ Department of Stomatology, \\ Jinan Central Hospital Affiliated to Shandong University, Jinan, Shandong 250014, P.R. China
}

Received July 9, 2013; Accepted August 20, 2013

DOI: $10.3892 /$ or.2013.2898

\begin{abstract}
The aim of the present study was to investigate the efficacy of using human umbilical cord blood-derived mesenchymal stem cells (hUCB-MSCs) as gene delivery vectors in the treatment of ovarian cancer. Lentivectors overexpressing cytosine deaminase (CD) and herpes simplex virus thymidine kinase (HSV-tk) (pGC-FU-CD-TK) were constructed, and confirmed by enzyme digestion, DNA sequence and western blotting. Quantitative PCR (PCR) was used to verify the overexpression of the fusion gene (CD and HSV-tk). SKOV3 cells were co-cultured with $\mathrm{MSCs} / \mathrm{tk}^{+} \mathrm{CD}^{+}$at a $1: 1$ ratio, and were then treated with the prodrugs $(\mathrm{GCV})$ and/or 5-fluorocytosine (5-FC) at different concentrations, and the cytotoxic effects were evaluated using MTT assay and flow cytometry. DNA sequencing demonstrated that the sequence of HSV-tk and $\mathrm{CD}$ genes were consistent with the objective sequence and western blotting verified that the constructed lentivector could produce the HSV-tk/CD gene. The packed titer was $2.00 \mathrm{e}+8 \mathrm{TU} / \mathrm{ml}$. The pGC-FU-CD-TK could be stably transferred to hUCB-MSCs, and the infection efficiency was almost $80 \%$. RT-PCR demonstrated that the expression levels of the $\mathrm{HSV}$-tk/CD fusion gene in $\mathrm{MSCs} / \mathrm{tk}^{+} \mathrm{CD}^{+}$group was 75 times that in the negative control $(\mathrm{P}<0.05)$. Compared with GCV or 5 -FC alone, the growth inhibition rate (GIR) was significantly higher in the combined treatment $(\mathrm{F}=85.35, \mathrm{P}<0.05)$. The reconstructed $\mathrm{MSCs} / \mathrm{tk}^{+} \mathrm{CD}^{+}$vectors were capable of slowing down the growth of human SKOV3 cells in the presence of prodrugs in vitro. The use of combination chemotherapy
\end{abstract}

Correspondence to: Dr Jingyan Jiang, Department of Gynecology, Jinan Central Hospital Affiliated to Shandong University, 105 Jiefang Road, Jinan, Shandong 250014, P.R. China

E-mail: jingyanejiang@yeah.net

Key words: mesenchymal stem cells, suicide cancer gene therapy, lentivector, SKOV3 cells exhibited a more significant inhibitory effect than using a single prodrug.

\section{Introduction}

Cancer is now believed to be a stem cell disease, therefore targeting cancer stem cells is one of the major goals in cancer treatment. Prodrug cancer gene therapy driven by mesenchymal stem cells (MSCs) may be one of several treatment modalities fulfilling these requirements. It represents an attractive tool for activating the prodrug directly within the tumor mass, thus avoiding systemic toxicity. In addition, MSCs lack major histocompatibility complex MHC-II and show only minimal MHC-I expression (1-3). The efficacy of suicide gene therapy relies on efficient gene delivery to tumors and the localized concentration of final gene products. MSCs possess tumor-tropic properties and have been consequently utilized to deliver extrogene for cancer treatment. These cells have a low immunogenic potential that makes them a unique tool to convert the relatively nontoxic prodrug into the highly toxic antitumor drug directly within the tumor mass. The increased production of inflammatory mediators found at the sites of a tumor is potentially responsible for recruitment and engraftment of MSCs (4). Tumor secreted chemotactic protein (MCP-1) has also been shown to be responsible for MSC migration to the tumor site (5). MSC-targeted gene therapy is a two-step process. In the first step, the gene for a foreign enzyme (bacterial, yeast or viral) is delivered and targeted to the tumor by transduced MSCs. In the second step, the enzymatic activity of gene product is able to convert a far less toxic prodrug to its cytotoxic substance at the tumor site. The prodrug produced by an enzymatic process within transduced MSCs kills neighboring tumor cells and also the more resistant cells in which it is formed.

In this study, we investigated the feasibility and efficacy of using hUCB-MSCs as gene delivery vectors for ovarian carcinoma gene therapy. We tested if these cells could be engineered to stably express the herpes simplex virus thymidine kinase gene (HSV-tk) and cytosine deaminase (CD) genes by 
using lentivirus gene transfer methods, whether they could exhibit tumor tropism and whether they could efficiently kill tumor cells when coupled with the HSV-tk/ganciclovir (GCV) and $\mathrm{CD} / 5$-fluorocytosine (5-FC) system.

\section{Materials and methods}

Materials. hUCB-MSCs (Shandong Province, umbilical cord blood stem cell bank) were sorted to obtain a homogenous $\mathrm{CD}_{105^{+}}$and CD24 population, and tested for purity by flow cytometry and for their ability to differentiate into osteogenic, chondrogenic and adipogenic lineages.

pWZLneoCDglyTK (donated by Professor Daoxin Ma, Central Laboratory, Qilu Hospital of Shandong University), pGC-FU vector $9955 \mathrm{bp}$, pGC-FU vector age I enzyme cutting production, SKOV3 cell line (Shanghai GeneChem Co., Ltd.).

\section{Methods}

Construction of double suicide gene overexpression lentivector ( $p G C-F U-C D-T K$ ). To acquire HSV-tk/CD genes, we used the plasmid pWZLneoCDglyTK as a molding board for polymerase chain reaction (PCR) amplification. The following primers were used for the PCR: forward primer, GAGGATCCCCGGGTACCGGTCG-CCACCATGTCGAA TAACGCTTTACAAAC and reverse primer, TCACCATGG TGGCG-ACCGGCCTTCCGGTATTGTCTCCTTC. Enzyme linearized the lentivirus vector (pGC-FU vector $9955 \mathrm{bp}$ ) and then directly connected them with objective genes and the production transformed into competent bacteria. Then PCR was used to identify the positive clone. The positive clone was picked and sequenced following which sequence analysis was performed. The following primers were used for the PCR and DNA sequencing: HSV-TK/CD fusion genes-SEQF, CATCT ACACCACACAACACC, ubiquitin-F, GGGTCAATATGTA ATTTTCAGTG and EGFP-N-R, CGTCGCCGTCCAGCTC GACCAG. The total PCR volume was $20 \mu 1: 12.4 \mu \mathrm{l}$ doublefiltered nanopure water, $0.4 \mu 1 \mathrm{HSV}-\mathrm{TK} / \mathrm{CD}$ fusion gene-SEQF primer $(10 \mu \mathrm{M}), 0.4 \mu 1$ EGFP-N-R primer $(10 \mu \mathrm{M}), 4 \mu 15 \mathrm{X}$ Taq buffer, $1.6 \mu \mathrm{l}$ DNTPs $(2.5 \mathrm{mM}), 1 \mu \mathrm{l}$ DNA template, $0.2 \mu \mathrm{l}$ Taq polymerase. Reactions were performed at $94^{\circ} \mathrm{C}$ for $2 \mathrm{~min}$, followed by 30 cycles at $94^{\circ} \mathrm{C}$ for $30 \mathrm{sec}, 60^{\circ} \mathrm{C}$ for $30 \mathrm{sec}$ and $72^{\circ} \mathrm{C}$ for $1 \mathrm{~min}$ and $72^{\circ} \mathrm{C}$ for $6 \mathrm{~min}$. The water was replaced as template to eliminate the possibility of exogenous nucleic acid contamination and false positive results. Using the automatic connection control transformant as template to eliminate non-specific amplification induced false positive results; using the positive control transformant as templates, amplification of part of the GAPDH gene, to troubleshoot PCR reagents, PCR instrument, and PCR reaction conditions caused false-negative results. The amplicons were verified by $1 \%$ agarose gel electrophoresis and western blotting was used to verify protein expression of objective gene after lentivirus transduction. The following antibodies were used for the western blotting: mouse anti-GFP and goat anti-mouse (Santa Cruz Biotechnology, Inc.) at a dilution ratio of 1:2,000.

Overexpression of lentivector, packaging and titration. Vectors were produced by standard transient transfection of a three-plasmid system into producer cells. Briefly, recombined expression plasmid pGC-LV, packaging plasmid pHelper 1.0

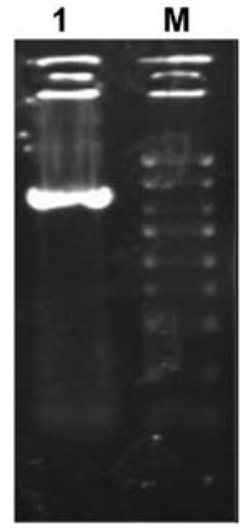

Figure 1. Gel electropherogram of PCR product for HSV-tk/CD gene. Lane 1, the product of HSV-tk/CD fusion genes (2518 bp); M, marker (5, 3, 2, 1.5, $1 \mathrm{~kb}$, and $750,500,250$ and $100 \mathrm{bp}$ ).

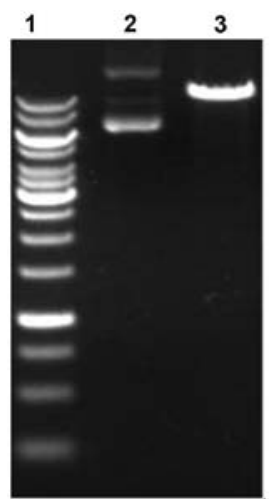

Figure 2. Agarose gel electrophoresis displays linear carrier pGC-FU vector existence. $1,1 \mathrm{~kb}$ marker $(10,8,6,5,4,3.5,3,2.5,2,1.5,1 \mathrm{~kb}$, and 750, 500 and $250 \mathrm{bp}$ ); 2, pGC-FU vector; 3, pGC-FU vector Age I Enzyme cutting product.

and envelope plasmid pHelper 2.0 were transferred into packaging cell line 293T using Lipofectamine 2000 transfection reagent (Invitrogen Life Technologies), according to the manufacturer's instructions. The transfection efficiency was observed through fluorescence microscopy and the lentivirus particles were observed by means of electron microscopy. High titer of lentivirus was harvested from the supernatant of virus producing cell culture and concentrated by high speed centrifugation with poly-l-lysine (PLL). Virus titer was determined by real-time quantitative PCR method. RNA was extracted using TRIzol (Invitrogen Life Technologies) according to the manufacturer's instructions. cDNA was synthesized according to the M-MLV (Promega Corporation) instructions. All manipulations were RNase-free. The determined recombinant plasmids were serially diluted 10 -fold in TE buffer, from $10^{-1}$ to $10^{-5}$. Each dilution was tested and used as amplification templates to construct curves by plotting the plasmid copy number logarithm against the $C_{t}$ values. All reactions were performed in triplicate and in at least 3 independent reactions, and the average relative content of pGC-FU-CD-TK transcripts was calculated using the $2^{-\Delta \Delta \mathrm{Ct}}$ method by iCycler IQ Multicolor Real-Time PCR Detection System (Bio-Rad, USA) procedure. SYBR master mixture was from Takara Bio, Inc. HSV-tk/CD fusion gene primer (forward primer, TGCTTCAGCCGCTACCC and reverse 


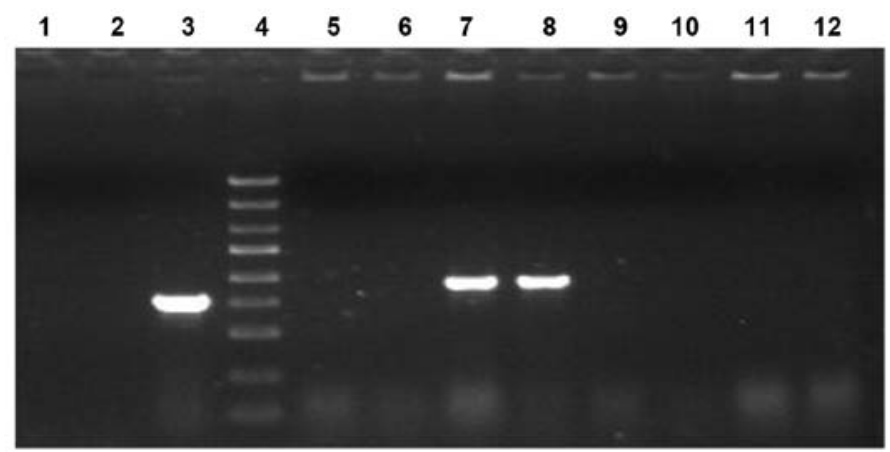

Figure 3. Identification of positive clones by PCR (941 bp). 1, Negative control ( $\left.\mathrm{ddH}_{2} \mathrm{O}\right) ; 2$, negative control (empty vector); 3, positive control (GAPDH); 4, marker (5, 3, 2, 1.5, $1 \mathrm{~kb}$, and 750, 500, 250 and $100 \mathrm{bp}) ; 5-12$, PCR products of recombinant plasmid pGC-FU-TK/CD DNA.

primer, AGTTCACCTTGATGCCGTTC). ACTIN primer (forward primer, GTGGACATCCGCAA-AGAC and reverse primer, AAAGGGTGTAACGCAACTA). The total volume of PCR protocol was $20 \mu \mathrm{l}$ : $10 \mu \mathrm{l}$ SYBR Premix Ex Taq, $1.0 \mu \mathrm{l}$ of each primer (5 $\mu \mathrm{M}$ each), $1 \mu \mathrm{l}$ DNA template, sterile water was added into the mixture to make $20 \mu \mathrm{l}$. Each run consisted of initial denaturation at $95^{\circ} \mathrm{C}$ for $15 \mathrm{sec}$ following by 40 consecutive cycles of denaturation at $95^{\circ} \mathrm{C}$ for $5 \mathrm{sec}$ and annealing at $60^{\circ} \mathrm{C}$ for $30 \mathrm{sec}$. The absorption values were read at the extended stage each time. Positive control (standard plasmid without dilution) and negative control (NTC) were added to each experiment, for quality control. Melting curve analysis was performed to verify the specificity of primers. Following PCR, denaturation at $95^{\circ} \mathrm{C}$ for $1 \mathrm{~min}$, then cooling to $55^{\circ} \mathrm{C}$ was performed to make DNA double chain to fully combine. From 55 to $95^{\circ} \mathrm{C}$, the temperature was increased by $0.5^{\circ} \mathrm{C}$ every step and the absorption value was read.

Transfection of hUCB-MSCs with lentivector co-expressing $C D$ and $H S V$ - $t k$. The hUCB-MSC cells have been shown to be easily infected with lentivirus in conditions where enhanced infection solution supplemented with $5 \mu \mathrm{g} / \mathrm{ml}$ polybrene were used. It has been determined that to obtain an $80 \%$ infection rate, the best multiplicity of infection (MOI) is 2 .

The third generation hUCB-MSC cells were seeded in 12-well plates at $1 \times 10^{6}$ cells/well and were randomly divided into 3 groups: objective gene infected, empty lentiviral vector and control group. MSCs were grown overnight and consecutively transduced with lentivectors for three days in a minimal volume of medium. Transgene expression was measured $72 \mathrm{~h}$ post-transduction by immunofluorescent staining. MSCs were harvested for analysis when fluorescence rate was $>80 \%$. Real-time PCR confirmed the integration and expression of extraneous gene. For RT-PCR assay, $10^{6}$ cells were used. GAPDH primer (forward primer, TGACTTCAACAGCGACACCCA and reverse primer, CAC CCTGTTGCTGTAGCCAAA) and double suicide gene primer (forward primer, AGCCTGGATGCCGAACAA and reverse primer, GCCTTCAAACAGCGTGCC) were used.

In vitro cytotoxicity assay of $\mathrm{MSCs} / \mathrm{tk}^{+} \mathrm{CD}^{+}$on $\mathrm{SKOV} 3$ cells. In order to observe if $\mathrm{MSCs} / \mathrm{tk}^{+} \mathrm{CD}^{+}$had any effect on ovarian cancer cell line SKOV3, they were co-cultured in a 24-well Transwell plate with a filter size of $0.4 \mu \mathrm{m}$. The upper chamber was seeded with $\mathrm{MSCs} / \mathrm{tk}^{+} \mathrm{CD}^{+}$, and the lower chamber was seeded with SKOV3 cells. MSCs/tk $\mathrm{CD}^{-}$were used as negative control and blank cells as positive controls. Twenty-four hours later, prodrug GCV/5-FC were added at different concentrations $(10 / 80,20 / 100,100 / 160 \mu \mathrm{g} / \mathrm{ml})$. MTT assay was performed to measure SKOV3 cell viability everyday up to 5 days and repeated three times. MSC viability was determined by standard MTT assay as previously described.

Apoptosis levels were assessed by using Annexin V-APC and PI dye kit (88-8007; eBioscience) according to the manufacturer's instructions. The data were acquired via flow cytometry (FCM) using a FACSCalibur system and analyzed using CellQuest acquisition software (BD Pharmingen).

Effect of prodrugs and $M S C / t k^{+} C D^{+}$on SKOV3 growth. Twenty-four-well Transwell plates with a diameter of $0.4 \mu \mathrm{m}$ were used to co-culture $\mathrm{MSCs} / \mathrm{tk}^{+} \mathrm{CD}^{+}$and SKOV3 cells. MSCs/ $\mathrm{tk}^{+} \mathrm{CD}^{+}$cells were plated in the upper compartment, while the lower compartment was plated with SKOV3 cells. GCV $(20 \mu \mathrm{g} / \mathrm{ml}), 5$-FC $(100 \mu \mathrm{g} / \mathrm{ml})$ or GCV/5-FC $(20 / 100 \mu \mathrm{g} / \mathrm{ml})$ were separately added to the co-culture after $24 \mathrm{~h}$. Cells were incubated for 5 days and growth inhibition rate (GIR) of SKOV3 cells was measured every other day by MTT assay and the experiment was repeated 3 times.

Statistical analysis. Statistical comparisons of groups were performed using SPSS 16.0 software. All data are presented as means $\pm \mathrm{SE}$. The statistical significance was determined by one-factor analysis of variance (ANOVA) with replication followed by the Holm-Sidak method. A P-value of $<0.05$ was considered to indicate statistically significant differences.

\section{Results}

Identification of the recombinant lentiviral plasmid $p G C$ $F U$-CD-TK vector. HSV-tk/CD genes were amplified by PCR using Taq DNA polymerase and agarose gel electrophoresis displayed HSV-tk/CD genes exist in the PCR product, as shown in Fig. 1. Age I Enzyme was used to cut the plasmid pGC-FU and the linear plasmid vector was recovered for agarose gel electrophoresis (Fig. 2). The linear lentivector was then fused with HSV-tk/CD gene and transferred into competent bacteria. PCR was used to identify the positive clone. The results showed that the 8th and 9th transformed clone successfully produced lentivector which include fusion gene of CD and HSV-TK (Fig. 3). DNA sequencing showed that the 


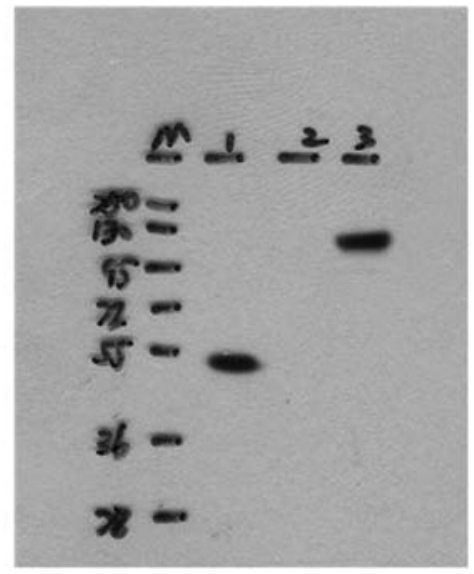

Figure 4. Western blotting showing fusion gene expression. M, marker; 1 WBSTD001 (48 kDa); 2, control (293T cells); 3, objective vector transfected $293 \mathrm{~T}$ cells, fusion gene $(91+28=119 \mathrm{kDa})$.

gene sequence of positive clone was consistent with objective sequence, identities $=898 / 898(100 \%)$. Western blotting was used to detect the transfected $293 \mathrm{~T}$ cells and we observed that HSV-TK/CD fused GFP gene was expressed in the objective plasmid. The size of inserted purpose fusion gene fragment in express cloning is $2472 \mathrm{bp}$. The above-mentioned PCR, DNA sequencing and western blotting demonstrated that we successfully constructed overexpression lentivector-pGC-FU-CD-TK, which included CD and HSV-TK fusion genes (Fig. 4).

Virus titer determination. Real-time quantitative reverse transcription polymerase chain reaction assay (qRT-PCR) has become the benchmark for detection and quantification of target gene expression level and have been utilized increasingly in the detection of viral load (Figs. 5 and 6). Melt curves are shown in Fig. 7, respectively. As a result, both presented a single peak. The consequences of melting curve were consistent with a single reaction product for each sample, that supported the high specificity of primers in another aspect. Pollution, primer dimer and nonspecific amplification were not observed.

The efficiency of lentivector in mediating gene transfer to MSCs. The GFP gene expression was observed by fluores-

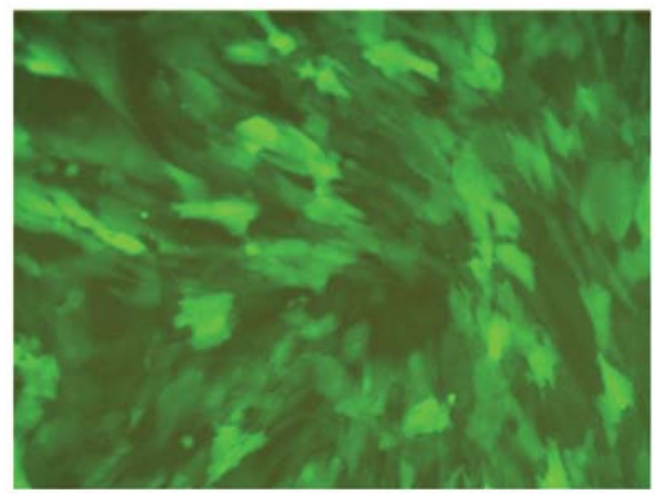

Figure 6. Fluorescent microscope image showing pGC-FU-CD-TK vector transfected hUCB-MSCs.

cent microscopy and, as shown in Fig. 6, 80-90\% cells had green fluorescence. The $\mathrm{C}_{\mathrm{t}}$ value showed the expression level of the fusion gene in MSC was constant and specific. RT-PCR demonstrated the expression level of the fusion gene in the $\mathrm{MSCs} / \mathrm{tk}^{+} \mathrm{CD}^{+}$group was 75 -fold that in the negative control group $(\mathrm{P}<0.05)$ (Fig. 7). These results indicate that $\mathrm{CD}$ and HSV-tk could be expressed efficiently and stably in hUCB-MSCs and lentivector is an ideal tool for effective gene transfer into hUCB-MSCs.

In vitro cytotoxicity assay of $\mathrm{MSCs} / \mathrm{tk}^{+} \mathrm{CD}^{+}$on $\mathrm{SKOV} 3$ cells. To study the effect of the MSCs/tk $\mathrm{CD}^{+}$on SKOV3 cell survival and proliferation, we co-cultured them and performed MTT and apoptosis assays. Our results indicated that SKOV3 cells were not sensitive to prodrugs in the negative control group and the blank control group when the concentration of GCV and 5-FC was 20/100 $\mu \mathrm{g} / \mathrm{ml}$. Notably, MTT assay revealed that cells co-cultured with $\mathrm{MSCs} / \mathrm{tk}^{+} \mathrm{CD}^{+}$showed a significant inhibition in proliferation $(\mathrm{p}<0.05)$ (Fig. 8A). Apoptosis assay also revealed that $\mathrm{MSCs} / \mathrm{tk}^{+} \mathrm{CD}^{+}$induced significantly higher apoptosis in SKOV3 cells compared to controls (Fig. 8B). Furthermore, at day 5, the GIR increased significantly in the presence of the prodrugs in a dose-dependent manner $(\mathrm{F}=27.31$, $\mathrm{P}<0.05$ ) (Fig. 9). These results indicate that cell viability of SKOV3 cells was significantly inhibited when co-cultured with $\mathrm{MSCs} / \mathrm{tk}^{+} \mathrm{CD}^{+}$.
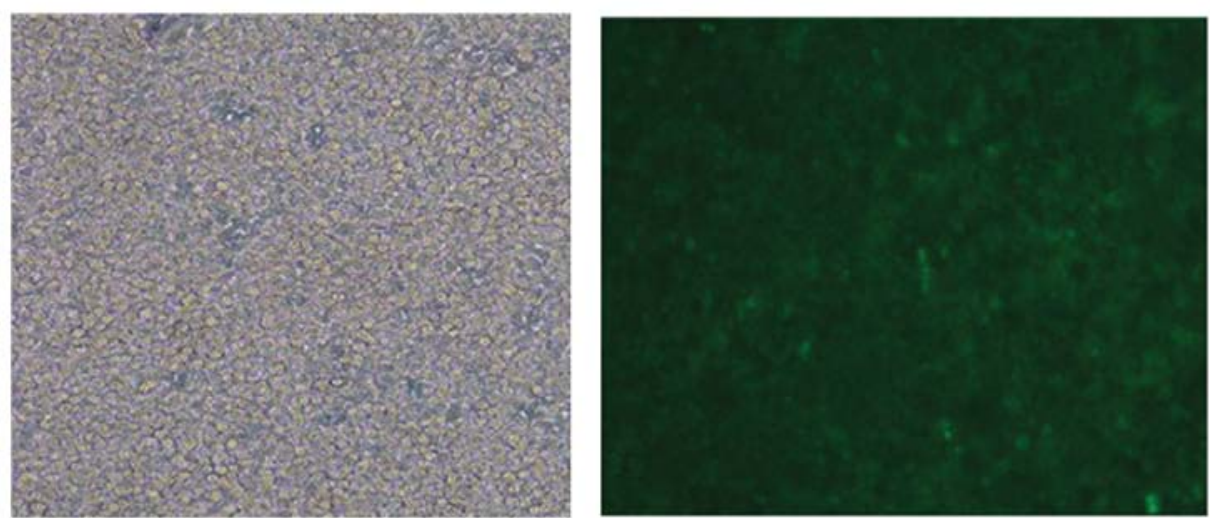

Figure 5. Objective virus (pGC-FU-CD-TK) infection 293T titer images; magnification, x100. 


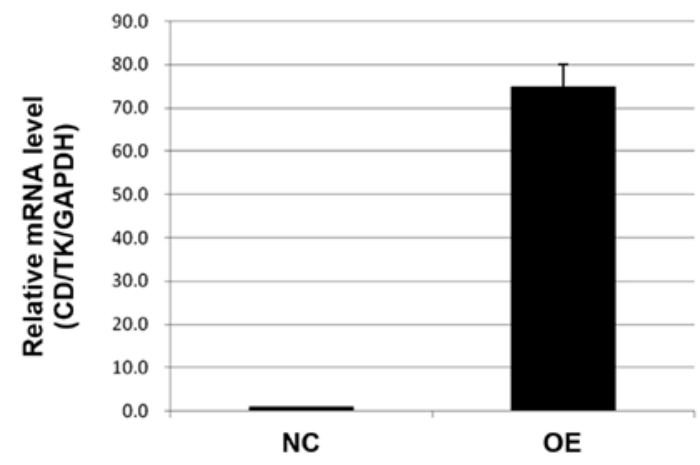

Figure 7. A histogram showing the expression level of HSV-tk/cd fusion gene in $\mathrm{MSCs} / \mathrm{tk}^{+} \mathrm{CD}^{+}$cells as determined by RT-PCR, $(\mathrm{P}<0.05)$. NC, negative control; OE.

A
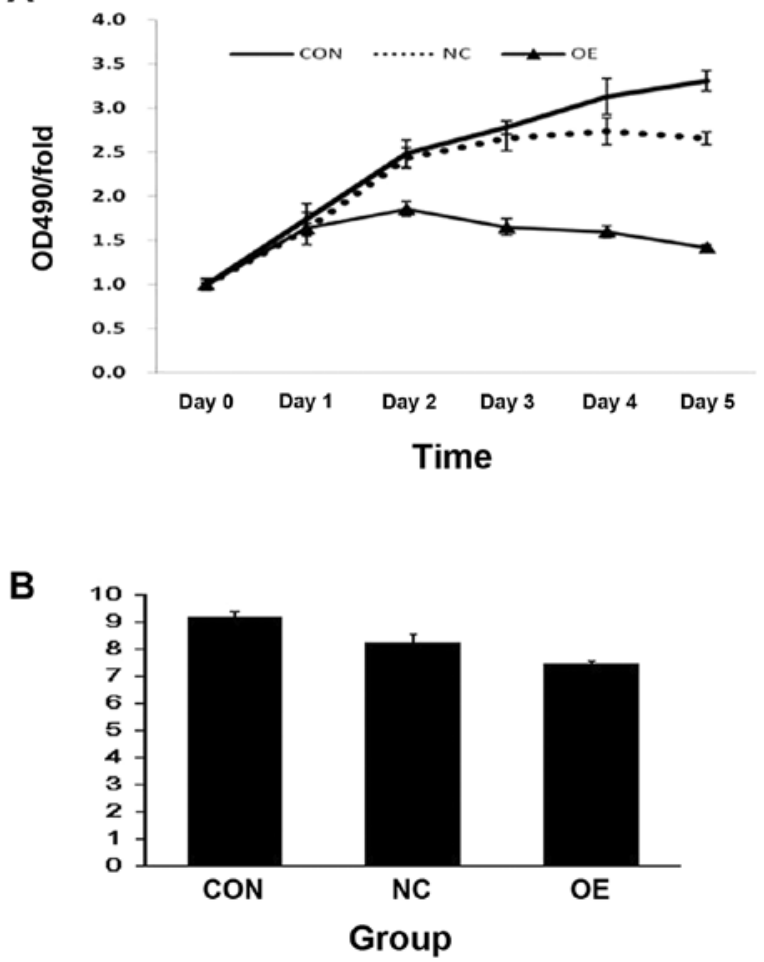

Figure 8. (A) SKOV3 cell viability tested by MTT assay and (B) apoptosis determined by FCM $(\mathrm{P}<0.05)$. CON, empty cells; NC, negative control (MSCs/tk/CD group); $\mathrm{OE}, \mathrm{MSCs} / \mathrm{tk}^{+} / \mathrm{CD}^{+}$group.

Effect of the prodrugs in combination on the growth rate of SKOV3 cells. To understand the effect of the prodrugs, in combination, on SKOV3 growth, we cultured the cells in the presence of either GCV or 5-FC alone or in combination. We found that there were no significant differences among the groups on day 1 and 3. However, there were significant differences between the groups on day 5. The GIRs were $7.00 \%$ in GCV group and $19.31 \%$ in the 5-FC group, respectively (Fig. 10). The GIR was $36.50 \%$, when the cells were cultured with both drugs together, and this was statistically significant $(\mathrm{F}=85.35, \mathrm{P}<0.05)$. This result suggested that the combined use of 2 prodrugs had stronger lethal effects than either one used alone.

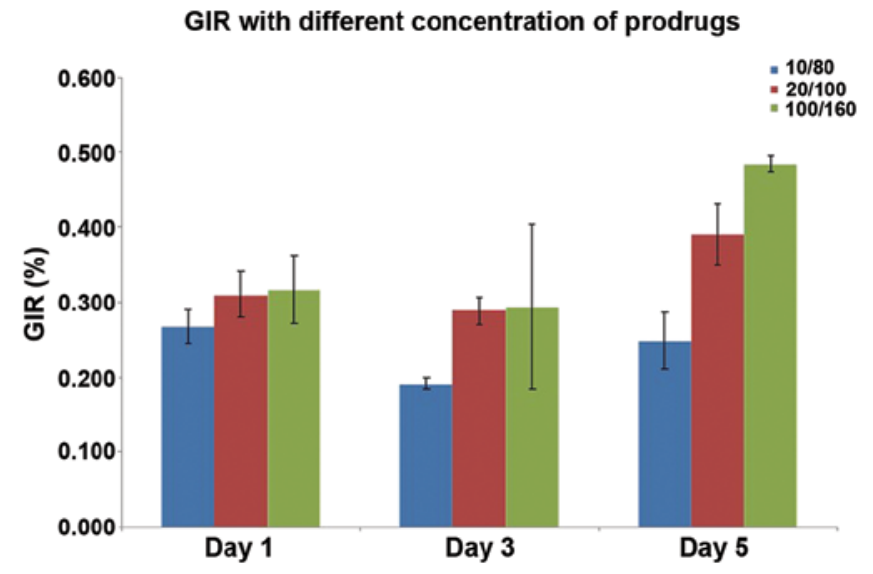

Figure 9. Histogram demonstrating the GIR differences between the different prodrug concentrations as determined by MTT assay. There were no significant differences between the groups on day 1 and 3, but there were significant differences between the groups on day $5(\mathrm{~F}=27.31, \mathrm{P}<0.01)$. GIR, growth inhibition rate.

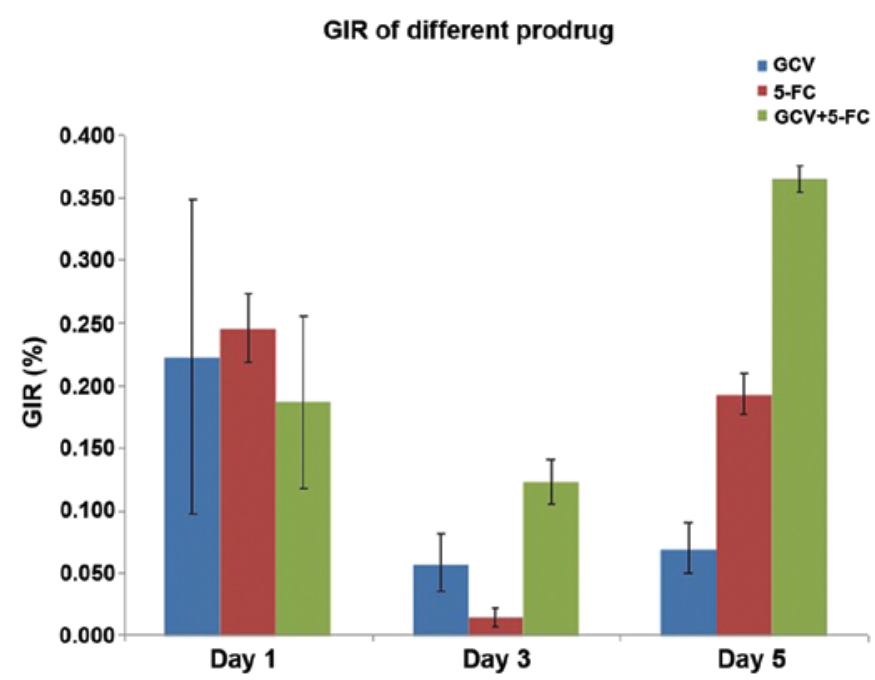

Figure 10. Histogram demonstrating the GIR differences between the GCV+5-FC, the GCV and the 5-FC group. There were no significant differences between the groups on day 1 and 3, but there were significant differences between the groups on day 5 . The GIR was $7.00 \%$ in the GCV group and $19.31 \%$ in the 5-FC group. Moreover, the survival rate was $36.5 \%$ when they were treated with the drugs simultaneously $(\mathrm{F}=85.35, \mathrm{P}<0.01)$. GIR, growth inhibition rate.

\section{Discussion}

Lentivectors are an attractive tool for gene transfer. The features of lentiviral vector such as infecting both dividing and non-dividing cells, holding larger segment size of exogenous gene, lower immune response, better safety, producing high titer of virus in host cells over long range, have made it popular in the current gene therapy research $(6,7)$. They have the ability to incorporate the target gene into the host chromosome, which forms the basis for stable gene expression for long periods of time (8). Thus, the efficiency of gene interference will not be lost with cell division. Another characteristic, which could be a distinct advantage, is that more rapid integration into the genome will reduce the potential for genetic mosaicism (9). 
We successfully constructed double suicide fusion gene overexpressing lentivector. It was determined by enzyme digestion, DNA sequence and western blotting that the fusion suicide gene was constructed with full function and integrity and expression of the double suicide gene.

Suicide gene therapy is an important strategy in cancer gene therapy. The optimization of vector system and the gene targeting transfer expression can increase the specific expression of suicide gene and enhance the effect of killing tumor cells. In recent years, considerable evidence has accumulated to suggest that MSCs have the potential to serve as vehicle to selectively deliver anticancer molecules to tumors, as they possess inherent tumor-tropic properties and can target tumor sites by migrating to and infiltrating bulk tumors in vivo $(10,11)$. MSCs exhibit strong tropism toward tumors that express receptors for chemokines and growth factors including SDF-1, MCP-1, HGF, IL-8, NT3, TGF-b and VEGF $(5,12-15)$. In this study, MSCs were engineered to produce the fusion gene by using lentiviral vector. Our results confirmed that hUCB-MSCs can be easily infected by the lentivector at a high efficiency of over $80.0 \%$. The hUCB-MSCs, express the target gene at a high efficiency and RT-PCR demonstrated that the expression level of $\mathrm{HSV}-\mathrm{tk} / \mathrm{cd}$ fusion gene in $\mathrm{MSCs} / \mathrm{tk}^{+} \mathrm{CD}^{+}$ was 75 -fold greater than in negative control $(\mathrm{P}<0.05)$. It is in this context that hUCB-MSCs have appeared to express exogenous gene efficiently, and could be one of the new target cells for genetic engineering. Furthermore, MSCs have immunosuppressive properties that may be useful in targeted gene therapy for sustained tissue specific engraftment. MSCs at the tumor site could continuously secrete antitumor factors, thereby protecting against drug induced organ specific (intestine, bladder and kidney) and hematological toxicities (16) and reducing the systemic drug adverse reactions. Lentiviral transduction does not cause the transduced MSCs to lose their basic stem cell identity (17). Our results suggest that hUCB-MSCs could be important in target gene transfer.

In this study, we tested the feasibility and efficacy of these therapeutic cells to function as cellular vehicles of double suicide gene in ovarian cancer therapy. We showed that hUCBMSCs could be a potential tool for ex vivo therapy in ovarian cancer. MSCs possess excellent migratory ability and exerted inhibitory effects on the proliferation of ovarian tumor cells. Gene-modification of MSCs with therapeutic genes clearly augmented their antitumor effect. Gene therapy employing MSCs as a targeting cytoreagent may be a promising approach.

Combined suicide gene therapies have recently emerged as an attractive alternative therapy for the treatment of various types of intractable cancer. CD/5-FC and HSV-TK/GCV system employed in our study, are the two most studied suicide systems. CD gene codes cytosine deaminase, an enzyme found in a variety of bacteria and fungi, also able to deaminate the nontoxic prodrug 5 -FC to its toxic metabolite 5 -fluorouracil (5-FU), which inhibits RNA and DNA synthesis during the $\mathrm{S}$ phase of the cell cycle. HSV-TK gene codes thymidine kinase, which is able to initially phosphorylate GCV, then subsequently incorporate into the host DNA and cause tumor cell death. Both mechanisms are similar, but the action segments are different $(18,19)$. Single suicide gene therapy has various limitations, drug resistance being the most common. The high incidence of tumor gene mutation is the foundation of drug resistance (20). Since different types of tumor cells have differences in sensitivity to different enzyme/prodrug systems, and tumor in vivo often exists in the form of a variety of clonogenic tumor cells, making it difficult to achieve radical tumor eradication by application of a single enzyme/prodrug system. This makes fusion gene therapy a valuable option in cancer treatment. It has been confirmed that HSV-TK has direct tumoricidal effects and cytosine deaminase (CD) has attracted considerable attention by virtue of its stronger bystander effects than other suicide genes and using them in a complementary fashion can achieve the best effect in killing tumor cells. Rogulski et al (21) showed that, when combined, these prodrug-converting enzymes were functionally superior to either single agent alone. Therefore, our study adopted the CDglyTK fusion gene, expecting to use these two systems with different functions to overcome the tumor cell type dependence in suicide gene therapy, and decrease the incidence of drug resistance. Our analysis showed that $\mathrm{MSCs} / \mathrm{tk}^{+} \mathrm{CD}^{+}$cells have a dual mode of action by both inhibiting cell growth and inducing apoptosis. MTT test showed that the GIR of SKOV3 cells when using GCV and 5-FC together, was significantly higher than that of either drug when used alone. These results showed that using double suicide gene therapy caused significant damage to tumor cells compared to a single suicide gene.

We also showed that the prodrug concentration is critical for the effectiveness of this therapy. When the concentration of the prodrugs increased, there was a significant inhibition of SKOV3 cells and this inhibition increased in a dose-dependent manner in vitro.

Previous studies $(22,23)$ have shown that genetically modified MSCs can inhibit tumor growth and prolong the lifespan of tumor-bearing animals. Transplantation of MSCs has been shown to suppress tumor growth by inhibiting Akt activation in a Kaposi's sarcoma model and to reduce the size of brain tumors and increase survival in a glioma model $(16,24)$. The bystander effect is believed to be mediated by gap junction intercellular cell-to-cell contacts such as Cx43. The bystander effect in suicide gene therapy is directly proportional to the degree of gap junction intercellular communication. It has been confirmed that MSCs and the human glioma cells can, through the gap between connection communication, promote bystander effect and kill tumor cells. On the other hand, due to the bystander effect of suicide gene, while they destroy cancer cells they also kill the MSCs, thereby reducing the long-term survival of MSCs $(18,22)$.

The specific mechanism of interaction between MSCs and tumor cells is not completely understood and this warrants further studies, including a possible MSC contribution to tumor stroma and vasculature, MSC-mediated antitumor immune suppression, and the potential malignant transformation of cultured MSCs (25-27). MSCs on tumor growth have two-way regulating functions, which reflects that MSCs have heterogeneity. Different subsets of the MSCs have differences in gene expression, which in turn leads to the expression of different types of proteins and chemokine receptors, which are not consistent. Nonetheless, we highlight the novel prospects of MSC-based tumor therapy, which appears to be a promising approach. While this strategy remains to be tested in-depth, including various orthotropic or metastatic tumor models, it may greatly improve the suicide gene treatment results. 


\section{Acknowledgements}

This study was supported by the Promotive Research Fund for young and middle-aged scientists of Shandong Province (no. 2006BS03019).

\section{References}

1. Le Blanc K: Immunomodulatory effects of fetal and adult mesenchymal stem cells. Cytotherapy 5: 485-489, 2003.

2. Koppula PR, Chelluri LK, Polisetti N and Vemuganti GK: Histocompatibility testing of cultivated human bone marrow stromal cells - a promising step towards pre-clinical screening for allogeneic stem cell therapy. Cell Immunol 259: 61-65, 2009.

3. Griffin MD, Ritter T and Mahon BP: Immunological aspects of allogeneic mesenchymal stem cell therapies. Hum Gene Ther 21: $1641-1655,2010$

4. Kidd S, Caldwell L, Dietrich M, et al: Mesenchymal stromal cells alone or expressing interferon-beta suppress pancreatic tumors in vivo, an effect countered by anti-inflammatory treatment. Cytotherapy 12: 615-625, 2010.

5. Dwyer RM, Potter-Beirne SM, Harrington KA, et al: Monocyte chemotactic protein-1 secreted in primary breast tumors stimulates migration of mesenchymal stem cells. Clin Cancer Res 13 : 5020-5027, 2007.

6. Barraza RA and Poeschla EM: Human gene therapy vectors derived from feline lentiviruses. Vet Immunol Immunopathol 123 23-31, 2008.

7. Barraza RA, Rasmussen CA, Loewen N, et al: Prolonged transgene expression with lentivectors in the aqueous humor outflow pathway of nonhuman primates. Hum Gene Ther 20: 191-200, 2009.

8. Pfeifer A and Hofmann A: Lentiviral transgenesis. Methods Mol Biol 530: 391-450, 2009.

9. Park F: Lentivectors: are they the future of animal transgenesis? Physiol Genomics 31: 159-173, 2007.

10. Nakamizo A, Marini F, Amano T, et al: Human bone marrowderived mesenchymal stem cells in the treatment of gliomas. Cancer Res 65: 3307-3318, 2005.

11. Xiang J, Tang J, Song C, et al: Mesenchymal stem cells as a gene therapy carrier for treatment of fibrosarcoma. Cytotherapy 11: 516-526, 2009

12. Khakoo AY, Pati S, Anderson SA, et al: Human mesenchymal stem cells exert potent antitumorigenic effects in a model of Kaposi's sarcoma. J Exp Med 203: 1235-1247, 2006.

13. Loebinger MR, Eddaoudi A, Davies D and Janes SM: Mesenchymal stem cell delivery of TRAIL can eliminate metastatic cancer. Cancer Res 69: 4134-4142, 2009.
14. Menon LG, Picinich S, Koneru R, et al: Differential gene expression associated with migration of mesenchymal stem cells to conditioned medium from tumor cells or bone marrow cells. Stem Cells 25: 520-528, 2007.

15. Chang DY, Yoo SW, Hong Y, et al: The growth of brain tumors can be suppressed by multiple transplantation of mesenchymal stem cells expressing cytosine deaminase. Int J Cancer 127: 1975-1983, 2010

16. Rustum Y, Cao S, Durrani F and Fakih M: Se-(methyl)selenocysteine (MSC) potentiates the antitumor activity of irinotecan against human tumor xenografts and protects against drug induced toxicity. J Clin Oncol 22 (Suppl 14): 2068, 2004.

17. Wang F, Dennis JE, Awadallah A, et al: Transcriptional profiling of human mesenchymal stem cells transduced with reporter genes for imaging. Physiol Genomics 37: 23-34, 2009.

18. Kucerova L, Altanerova V, Matuskova M, et al: Adipose tissuederived human mesenchymal stem cells mediated prodrug cancer gene therapy. Cancer Res 67: 6304-6313, 2007.

19. Amano S, Li S, Gu C, et al: Use of genetically engineered bone marrow-derived mesenchymal stem cells for glioma gene therapy. Int J Oncol 35: 1265-1270, 2009.

20. Huang SY, Zhang DS, Han JQ, et al: Radiosensitization and anti-tumour effects of cytosine deaminase and thymidine kinase fusion suicide gene in human adenoid cystic carcinoma cells. J Int Med Res 37: 479-490, 2009.

21. Rogulski KR, Wing MS, Paielli DL, et al: Double suicide gene therapy augments the antitumor activity of a replicationcompetent lytic adenovirus through enhanced cytotoxicity and radiosensitization. Hum Gene Ther 11: 67-76, 2000.

22. Cavarretta IT, Altanerova V, Matuskova M, et al: Adipose tissuederived mesenchymal stem cells expressing prodrug-converting enzyme inhibit human prostate tumor growth. Mol Ther 18: 223-231, 2010.

23. Uchibori R, Okada T, Ito T, et al: Retroviral vector-producing mesenchymal stem cells for targeted suicide cancer gene therapy. J Gene Med 11: 373-381, 2009.

24. Mori K, Iwata J, Miyazaki M, et al: Bystander killing effect of tymidine kinase gene-transduced adult bone marrow stromal cells with ganciclovir on malignant glioma cells. Neurol Med Chir 50: 545-553, 2010.

25 Bagley RG, Weber W, Rouleau C, et al: Human mesenchymal stem cells from bone marrow express tumor endothelial and stromal markers. Int J Oncol 34: 619-627, 2009.

26. Mishra PJ, Mishra PJ, Humeniuk R, et al: Carcinoma-associated fibroblast-like differentiation of human mesenchymal stem cells. Cancer Res 68: 4331-4339, 2008.

27. Yang SH, Park MJ, Yoon IH, et al: Soluble mediators from mesenchymal stem cells suppress $\mathrm{T}$ cell proliferation by inducing IL-10. Exp Mol Med 41: 315-324, 2009. 